AperTO - Archivio Istituzionale Open Access dell'Università di Torino

\title{
Generalized Performance Ratios and Risk Optimization
}

\section{This is the author's manuscript}

Original Citation:

Availability:

This version is available http://hdl.handle.net/2318/1591171

since 2018-04-05T10:16:22Z

Published version:

DOI:dx.doi.org/10.12988/ams.2016.66196

Terms of use:

Open Access

Anyone can freely access the full text of works made available as "Open Access". Works made available under a Creative Commons license can be used according to the terms and conditions of said license. Use of all other works requires consent of the right holder (author or publisher) if not exempted from copyright protection by the applicable law. 
Applied Mathematical Sciences, Vol. 10, 2016, no. 55, 2709 - 2726

HIKARI Ltd, www.m-hikari.com

http://dx.doi.org/10.12988/ams.2016.66196

\title{
Generalized Performance Ratios and Risk Optimization
}

\author{
Christos E. Kountzakis \\ Department of Mathematics \\ University of the Aegean \\ 83200 Samos Greece \\ Luisa Tibiletti \\ Dipartimento di Management \\ University of Torino-Torino, 10134 Italy \\ Simone Farinelli \\ Core Dynamics GmbH, Zurich 8006 Switzerland
}

Copyright (c) 2016 Christos E. Kountzakis, Luisa Tibiletti and Simone Farinelli. This article is distributed under the Creative Commons Attribution License, which permits unrestricted use, distribution, and reproduction in any medium, provided the original work is properly cited.

\begin{abstract}
In this paper, we generalize the notion of the performance measure by using a variety of coherent risk measures. We prove that these classes of coherent risk measures assure the properties of the acceptability indexes. In separate sections classes associated to Expected Shortfall and Shortfall Risk are examined both with their sensitivity, and also the general static optimization problem of these ratios is studied.
\end{abstract}

Mathematics Subject Classifications: 46B40, 91G40

Keywords: coherent risk measures; performance ratios; optimization 


\section{Introduction}

In the present paper, we extend the notion of the performance ratios, by using a variety of coherent risk measures and pricing functionals of a portfolio under a static framework. Specifically, we suppose that the space $E$ in which the "tomorrow" value variables of a portfolio lie in, is a subspace of some $L^{0}(\Omega, \mathcal{F}, \mathbb{P})$, where $(\Omega, \mathcal{F}, \mathbb{P})$ is a non-atomic probability space which describes the uncertainty "today". $E$ is some partially ordered normed space. It is well-known that a seminal paper on the topic of the acceptability indexes is [4]. In the present paper, the properties of the performance ratios considered, are compared to the properties of the acceptability indexes considered in [4]. Specifically, the main properties are: monotonicity, scale -invariance, arbitrageconsistency, concavity and Fatou continuity. About the risk measures being used in the paper for the performance ratio formulation, are actually general coherent risk measures defined on ordered dual systems consisted by reflexive and sepcifically non -reflexive spaces and also the expected shortfall and risk measures related to shortfall risk. The presence of non-reflexive spaces is important due to the fact that $E S_{a}$ is defined on $L^{1}$. The sesitivity of performance ratios being built on coherent isk measures relying on Expected Shortfall, such as spectral risk measues is also studied in a separate section. The general problem of the maximization of a performance ratio over a set of not necessarily convex set of constraints is also solved under interior point methods and for $L^{2}$-spaces. This is also the way that the main content of the paper is organized. Finally, the Appendix is separated into the first part, in which the basics about strictly positive functionals in spaces with the Riesz Decomposition Property are mentioned. In the second part, the weak compactness of the representation set of the Expected Shortfall functionals' is deduced, while in the third part of the Appendix the basics about the Henig Dilating Cones are mentioned -which are used in the section regarding the optimization problem. In financial practice, RAROC is a performance ratio, which is widely used. The results of the present paper generalize the RAROC, especially in the form which expresses the ratio of the expected value of some financial position divided by the economic capital of it. In this exact form, may by equal to

$R A R O C(x)=\frac{\mathbb{E}(x)}{\rho(x)}$, where $\rho$ corresponds to some coherent risk measure, or in a better way to the Expected Shortfall $E S_{a}$.

\section{General Performance Ratios}

We consider the ordered Banach space $E$, a cone with interior points $P$ in $E$ and $x_{0} \in$ int $P$. We also consider the coherent risk measure $\rho$ with respect to $P$ and $x_{0}$ :

$$
\rho(y)=\inf \left\{m \in \mathbb{R} \mid y+m \cdot x_{0} \in P\right\}
$$


and also a subspace $G$ of $E$. We also suppose that 0 belongs to the $\sigma\left(E^{*}, G\right)$ closure of the base $B_{x_{0}}$ of $P^{0}$, then by [15, Cor.13], $G$ has the property that $\rho(G) \geq 0$. Hence we may define the following performance measure:

Definition 1. $a_{\rho, f}: E \rightarrow \mathbb{R} \cup\{\infty\}$, such that

$$
a_{\rho, f}(x)=\frac{f(x)}{\rho(x)},
$$

where $f$ is some strictly positive functional of $E$.

Notice that the division $\frac{c}{0}=\infty$, if $c>0$ and 0 is a result of a calculation on some positive variable.

Definition 2. $a_{\rho, f}$ is called arbitrage consistent with respect to $G$, if for any $x \in P \cap G, a_{\rho, f}(x)=\infty$.

Theorem 3. $a_{\rho, f}$ scale invariant, monotone and arbitrage-consistent with respect to $G$.

Proof: For abbreviation we denote $a_{\rho, f}$ by $a$. We also take some $t \in \mathbb{R}_{+}$.

(i) For the Monotonicity Property, if $x \leq y$ with respect to the partial ordering which is defined by the cone $P$ on $E$, and $\rho(x) \rho(y)>0, \rho(y) \leq$ $\rho(x)<0$ or $0<\rho(y) \leq \rho(x)$. This implies $\frac{f(x)}{\rho(x)} \leq \frac{f(y)}{\rho(y)}$. For the case where $\rho(x) \rho(y)<0$ the proof is analogous. Finally,

$$
a(x)=\frac{f(x)}{\rho(x)} \leq \frac{f(x)}{\rho(y)} \leq \frac{f(x)}{\rho(x)}=a(y) .
$$

(ii) Since $f$ is strictly positive, $f(\lambda \cdot x)=\lambda \cdot f(x)$, and $\rho(\lambda \cdot x)=\lambda \cdot \rho(x)$ since $\rho$ is coherent. Then, $a$ is scale invariant.

(iii) If $x \in P \backslash\{0\}, f(x)>0$ and $\rho(x) \geq 0$, because $x \in G$. On the other hand, $\rho(x) \leq 0$, because $x \in P$. Finally, $\rho(x)=0$ and $a(x)=\infty$.

Theorem 4. We assume some $t \in \mathbb{R}_{+}$. If $f, \rho$ take positive values, then $a_{\rho, f}$ is a concave function, if $\rho$ is a convex risk measure.

Proof: If $\rho(\lambda x+(1-\lambda) y) \leq \lambda \rho(x)+(1-\lambda) \rho(y), \lambda \in(0,1), x, y \in E$, and these values are positive, this implies $\frac{1}{\rho(\lambda x+(1-\lambda) y)} \geq \frac{1}{\lambda \rho(x)+(1-\lambda) \rho(y)}$ and moreover

$$
\frac{f(\lambda x+(1-\lambda) y)}{\rho(\lambda x+(1-\lambda) y)} \geq \frac{\lambda f(x)+(1-\lambda) f(y)}{\lambda \rho(x)+(1-\lambda) \rho(y)}=a(x, y)
$$


$a(x, y) \geq \lambda \frac{f(x)}{\rho(x)}+(1-\lambda) \frac{f(y)}{\rho(y)}$. If $\frac{f(x)}{\rho(x)}, \frac{f(y)}{\rho(y)} \geq t$, then $\lambda \frac{f(x)}{\rho(x)}+(1-\lambda) \frac{f(y)}{\rho(y)} \geq t$ and this finally implies

$$
\frac{f(\lambda x+(1-\lambda) y)}{\rho(\lambda x+(1-\lambda) y)} \geq t
$$

Proposition 5. For each coherent risk measure of the form $\rho(x)=\sup _{\pi \in D} \pi(-x), \pi \in$ $E_{+}^{0}$, we have

$$
a_{\rho, f}(x) \leq \inf \left\{\frac{-f(x)}{\pi(x)}, \pi \in D\right\}
$$

where if $D$ is $\sigma\left(E^{*}, E\right)$-compact, the ${ }^{\prime} \leq^{\prime}$ in this representation becomes ${ }^{\prime}={ }^{\prime}$.

Proof: $\frac{f(x)}{\rho(x)}=\frac{f(x)}{\sup _{\pi \in D} \pi(-x)} \leq \inf \left\{\frac{f(x)}{\pi(-x)}, \pi \in D\right\}$. If $D$ is $\sigma\left(E^{*}, E\right)$-compact, then $\rho(x)=\pi_{x}(-x), \pi_{x} \in D$. Then, in the above inequality, equality holds.

\section{Performance Ratios on $L^{p}$ Spaces}

\subsection{Expected -Shortfall Based Perfomance Ratios}

First of all, we assume a probability space $(\Omega, \mathcal{F}, \mathbb{P})$. Looking back at the seminal article on Performance Measures -[4] and specifically going to [4, Th.1] of Section 2.2, we notice that a possible family of $\mathcal{D}_{x}, x \in \mathbb{R}_{+}$can be

$$
\mathcal{D}_{x}=\left\{Q \mid Q<<P, 0 \leq \frac{d Q}{d P} \leq x\right\}, x \in \mathbb{R}_{+} .
$$

The family $\mathcal{D}_{x}, x \in \mathbb{R}_{+}$may define the acceptability index

$$
\alpha_{\mathcal{D}}(x)=\sup \left\{x \in \mathbb{R}_{+} \mid \inf _{Q \in \mathcal{D}_{x}} \mathbb{E}^{Q}(x) \geq 0\right\} .
$$

The functional $\rho_{x}(x)=-\inf _{Q \in \mathcal{D}_{x}} \mathbb{E}^{Q}(x), x \in \mathbb{R}_{+}$, is related to the Expected Shortfall if it is defined on $L^{1}(\Omega, \mathcal{F}, \mathbb{P})$, due to the dual representation of the Expected Shortfall, see in [11, Th.4.1]. Specifically,

$$
\rho_{x}(x)=E S_{\frac{1}{x}}(x), x \in L^{1}(\Omega, \mathcal{F}, \mathbb{P}), x \in \mathbb{R}_{+} .
$$

The existence of the extreme measure $Q_{x}^{*}(x)$ is implied in this case by the weak- star compactness of the order-interval $[0, x \mathbf{1}]$. The relevant cones $\mathcal{A}_{x}$ may be defined as

$$
\mathcal{A}_{x}=\left\{x \in L^{1} \mid \rho_{x}(x) \leq 0\right\},
$$

either via, or without Expected Shortfall. As a consequence

$$
\alpha(x)=\sup \left\{x \in \mathbb{R}_{+} \mid x \in \mathcal{A}_{x}\right\} .
$$

For a detailed proof of the above, see Appendix 6.1. 
Definition 6. We define the Expected Shortfall Ratio of the cash flow $x \in L^{1}(\Omega, \mathcal{F}, \mathbb{P})$, as follows: $E S R_{t}(x)=\frac{\mathbb{E}(x)}{E S_{t}(x)}$ if $\mathbb{E}(x)>0$, and $E S R_{t}(x)=0$ otherwise, where $t \in(0,1)$.

Theorem 7. ESR $R_{t}$ is monotone, scale-invariance, and law invariance.

Proof: Except the law-invariance, which is a consequence of the Expected Shortfall, the proof of the other arguments arises from Theorem 3.

Theorem 8. ESR $R_{t}$ is concave.

Proof: It arises from the Theorem 4.

Theorem 9. ESR $R_{t}$ satisfies the Fatou property, probably passing to subsequences.

Proof: If some sequence $x_{n}$ converges to $x$ in $\mathbb{P}$, then there exists some subsequence $x_{k_{n}} \rightarrow x, \mathbb{P}$-a.e. This implies $\mathbb{E}\left(x_{k_{n}}\right) \rightarrow \mathbb{E}(x)$ and $E S_{t}\left(x_{k_{n}}\right) \rightarrow$ $E S_{t}(x)$, since $E S_{t}$ is Lipschitz continuous with respect to $\|\cdot\|_{1}$-norm. Hence $E S R_{t}\left(x_{k_{n}}\right) \geq x$, if $E S_{t}(x) \neq 0$.

Theorem 10. ESR $R_{t}$ is arbitrage-consistent with respect to any solid subspace of $L^{1}(\Omega, \mathcal{F}, \mathbb{P})$.

Proof: If $x>0, \mathbb{P}-$ a.e. then since $E S_{t}$ is coherent, $E S_{t}(x) \leq 0$. If $x$ is an element of any solid subspace $S$ of $L^{1}((\Omega, \mathcal{F}, \mathbb{P})$, then from Theorem [15, Th.11], $E S_{t}(x) \geq 0 x \in S$, hence $E S_{t}(x)=0, x \in S \cap L_{+}^{1}(\Omega, \mathcal{F}, \mathbb{P})$. Also $\mathbb{E}(x)>0$ in this case, hence $\frac{\mathbb{E}(x)}{E S_{t}(x)}=\infty$.

\subsection{Performance ratios related to coherent risk mea- sures in $L^{p}$ spaces}

In [10, Th.1.1], the dual representation of any continuous coherent risk measure $\rho: L^{p} \rightarrow \mathbb{R}\left(\frac{1}{p}+\frac{1}{q}=1\right.$, where if $p=1$, then $q=\infty$ is given by

$$
\rho(x)=\sup _{g \in G} \mathbb{E}((-x) g),
$$

where $G \subseteq L_{+}^{q}$, such that $\sup _{g \in G}\|g\|_{q}<\infty$, and $\mathbb{E}(g)=1$ for any $g \in G$. This Theorem denotes both by Krein $-\hat{S}$ mulian Theorem, that the sup remains the same, if we take the set $K=\overline{c o}(G)$. Then the set $G$ may be assumed to be convex, closed and norm-bounded, hence weakly (or weak -star, in the case of $\left.L^{\infty}\right)$ compact. The performance ratio $a_{\rho, f}$, where $\rho(x)=\sup _{g \in K} \mathbb{E}((-x) g)$ and $f$ is a strictly positive, continuous functional of $L_{+}^{p}$, has the following properties: 
Theorem 11. $a_{\rho, f}$ is concave, scale-invariant and monotone. Also, any $a_{\rho, f}$ satisfies the Fatou property.

Proof: The concavity is implied by Theorem 4. For the scale invariance $\frac{f(\lambda x)}{\rho(\lambda x)}=\frac{\lambda f(x)}{\lambda \rho(x)}=\frac{f(x)}{\rho(x)}, x \in L^{p}, \lambda \in \mathbb{R}_{+}$. If $x \geq y$ and $\rho(x) \rho(y)>0, \rho(y) \leq$ $\rho(x)<0$ or $0<\rho(y) \leq \rho(x)$. This implies $\frac{f(x)}{\rho(x)} \leq \frac{f(y)}{\rho(y)}$. For the case where $\rho(x) \rho(y)<0$ the proof is analogous. Suppose that $a_{\rho, f}\left(x_{n}\right) \geq t, t>0$. If the sequence $x_{n}$ converges to $x$ in $\mathbb{P}$, then there exists some subsequence $x_{k_{n}} \rightarrow x$, $\mathbb{P}$-a.e. This implies $x_{k_{n}} \stackrel{L^{p}}{\rightarrow} x$ and $\rho\left(x_{k_{n}}\right) \rightarrow \rho(x)$, since $\rho$ is Lipschitz continuous with respect to $\|\cdot\|_{p}$-norm. Hence $a_{\rho, f}(x) \geq t$, if $\rho(x)>0$.

\subsection{Performance ratios related to Shortfall Risk}

According to the recent literature on elicitable risk measures, see [3], and their relation to shortfall risk, mentioned widely in [8, Sect.3]. Consider some increasing, convex loss function $\ell: \mathbb{R} \rightarrow \mathbb{R}$ and the expected loss $\mathbb{E}(\ell(-x))$ if $x$ lies in the space $L^{\infty}(\Omega, \mathcal{F}, \mathbb{P})$.

Definition 12. The Expected Loss Ratio, associated to the loss fucntion $\ell$ is defined as follows:

$$
E L R_{\ell}(x)=\frac{\mathbb{E}(x)}{\mathbb{E}(\ell(-x))},
$$

if $\mathbb{E}(x)>0$, while $E L R_{\ell}(x)=0$, if $\mathbb{E}(x)=0$.

Theorem 13. $E L R_{\ell}$ is monotone and law invariance if $\ell$ is continuous and strictly increasing, either on $(-\infty, \epsilon)$ or on $(\epsilon,+\infty)$ for some $\epsilon>0$.

Proof: If $x \leq y, \mathbb{P}$-a.e., then $\mathbb{E}(x) \leq \mathbb{E}(y)$. Since $\ell$ is increasing and $-y \leq$ $-x$, then $\mathbb{E}(\ell(-y)) \leq \mathbb{E}(\ell(-x))$. Then $\frac{1}{\mathbb{E}(\ell(-x))} \leq \frac{1}{\mathbb{E}(\ell(-y))}$, hence $\frac{\mathbb{E}(x)}{\mathbb{E}(\ell(-x))} \leq$ $\frac{\mathbb{E}(x)}{\mathbb{E}(\ell(-y))} \leq \frac{\mathbb{E}(y)}{\mathbb{E}(\ell(-y))}$. From $[3$, Def.4.2], the shortfall measure being defined by $\ell$ as

$$
\rho_{\ell}(F)=\inf \left\{x \in \mathbb{R} \mid \int_{\mathbb{R}} \ell(-y-x) d F(y) \leq x_{0}\right\}
$$

is law invariance since by [8] $\ell$ is continuous, the value $\rho_{\ell}(F)$ on the distribution $F$ is the solution of the equation

$$
\int_{\mathbb{R}} \ell\left(-y-\rho_{\ell}(F)\right) d F(y)=x_{0}
$$

According to the recent leterature on elicitable risk measures, see [3], and their relation to shortfall risk, mentioned widely in [8, Sect.3]. Consider some increasing, convex loss function $\ell: \mathbb{R} \rightarrow \mathbb{R}$ and the expected loss $\mathbb{E}(\ell(-x))$ if $x$ lies in the space $L^{\infty}(\Omega, \mathcal{F}, \mathbb{P})$. 
Definition 14. The Expected Loss Ratio, associated to the loss fucntion $\ell$ is defined as follows:

$$
E L R_{\ell}(x)=\frac{\mathbb{E}(x)}{\mathbb{E}(\ell(-x))},
$$

if $\mathbb{E}(x)>0$, while $E L R_{\ell}(x)=0$, if $\mathbb{E}(x)=0$.

Theorem 15. ELR $R_{\ell}$ is monotone and law invariance if $\ell$ is continuous and strictly increasing, either on $(-\infty, \epsilon)$ or on $(\epsilon,+\infty)$ for some $\epsilon>0$.

Proof: If $x \leq y, \mathbb{P}$-a.e., then $\mathbb{E}(x) \leq \mathbb{E}(y)$. Since $\ell$ is increasing and $-y \leq$ $-x$, then $\mathbb{E}(\ell(-y)) \leq \mathbb{E}(\ell(-x))$. Then $\frac{1}{\mathbb{E}(\ell(-x))} \leq \frac{1}{\mathbb{E}(\ell(-y))}$, hence $\frac{\mathbb{E}(x)}{\mathbb{E}(\ell(-x))} \leq$ $\frac{\mathbb{E}(x)}{\mathbb{E}(\ell(-y))} \leq \frac{\mathbb{E}(y)}{\mathbb{E}(\ell(-y))}$. From $[3$, Def.4.2], the shortfall measure being defined as

$$
\left.\int \mathbb{R} \ell(-y-x) d F(y) \leq x_{0}\right\}
$$

is law invariance since by [8] $\ell$ is continuous, the value $\rho_{\ell}(F)$ on the distribution $F$ is the solution of the equation

$$
\int_{\mathbb{R}} \ell\left(-y-\rho_{\ell}(F)\right) d F(y)=x_{0}
$$

Theorem 16. ELR $R_{\ell}$ satisfies the Fatou property, probably passing to subsequences, if $\ell$ is continuous and strictly increasing, either on $(-\infty, \epsilon)$ or on $(\epsilon,+\infty)$ for some $\epsilon>0$.

Proof: If some sequence $x_{n}$ converges to $x$ in $\mathbb{P}$, then there exists some subsequence $x_{k_{n}} \rightarrow x, \mathbb{P}$-a.e. This implies $\mathbb{E}\left(x_{k_{n}}\right) \rightarrow \mathbb{E}(x)$ and $E L R_{\ell}\left(x_{k_{n}}\right) \rightarrow$ $E L R_{\ell}(x)$, since $\frac{1}{\ell\left(x_{k_{n}}\right)} \rightarrow \frac{1}{\ell(x)}$. If $\mathbb{E}(\ell(x)) \neq 0$, and $\frac{\mathbb{E}\left(x_{k_{n}}\right)}{\mathbb{E}\left(\ell\left(x_{k_{n}}\right)\right)} \geq x$, then $\frac{\mathbb{E}(x)}{\mathbb{E}(\ell(x))} \geq x$.

\section{Strong Sensitivity of Performance Ratios}

We recall the following Definition of Strict Sensitivity obtained by [9]:

Definition 17. $\rho$ is called strictly sensitive if $x \geq x^{\prime}, \mu$-a.e., $\rho(x)=\rho\left(x^{\prime}\right) \Rightarrow$ $x=x^{\prime}, \mu-a . e$.

We also need another notion of sensitivity on $L^{1}$ :

Definition 18. A monetary risk measure $\rho$ on $L^{1}$ is strongly sensitive, if and only if $x=y, \mu$-a.e. $\Rightarrow \rho(x)=\rho(y)$. 
Also, the sensitivity of a peformance ratio $a_{\rho, f}$ may be defined in an equivalent way:

Definition 19. A performance ratio $a_{\rho, f}$ on $L^{1}$ is strongly sensitive, if and only if $x=y$, -a.e. $\Rightarrow a_{\rho, f}(x)=a_{\rho, f}(y)$.

Theorem 20. For any $a \in(0,1]$, the Expected Shortfall ES $S_{a}$ is strongly sensitive.

Proof: By the dual representation theorem of Expected Shortfall [?, Th.4.1], we have

$$
E S_{a}(x)=\max _{\pi \in\left[0, \frac{1}{a} \mathbf{1}\right]} \pi(-x)
$$

such that $\pi$ is a Radon -Nikodym derivative of some probability measure $Q_{\pi}<<\mu$,

$$
E S_{a}(|x|) \leq \frac{1}{a}\|x\|_{1}
$$

which implies that $E S_{a}$ is strongly sensitive.

Theorem 21. For any $a \in(0,1], b>1$ such that $\frac{1}{b}<\frac{1}{a}$, the Adjusted Expected Shortfall is strongly sensitive.

Proof: By [16, Lem.6] regarding the dual representation of Adjusted Expected Shortfall, we get that

$$
A E S_{a, b}(|x|)=\frac{1}{b}\|x\|_{1}
$$

hence the specific risk measure is strongly sensitive on $L^{1}$.

Theorem 22. Any spectral risk measure of the form $M_{m}(x)=\int_{0}^{1} a E S_{a}(x) d m(a)$ defined on $L^{1}$ is strongly sensitive.

Proof: By [1, Th.2.5], any spectral risk measure $M_{m}$ is a continuous, coherent risk measure on $L^{1}$, since $\int_{0}^{1} a d m(a)=1$ and $E S_{a}$ is a continuous, coherent risk measure on $L^{1}$. The coherence of $M_{m}$ is implied by [1]. The continuity of $M_{m}$ is implied by relation (6) in [1]. More specifically,

$$
\left|M_{m}(|x|)\right|=\int_{0}^{1} E S_{a}(|x|) a d m(a) \leq \frac{1}{b}\|x\|_{1} \cdot \frac{1}{b},
$$

for some $b>1$ such that $0<a \leq 1<b$.

Theorem 23. The pointwise limit of spectral risk measures on $L^{1}$ under the same measure of risk spectrum $m$ is a srongly sensitive coherent risk measure. 
Proof: If

$$
\rho(x)=\lim _{n} \int_{0}^{1} E S_{a_{n}}(x) a_{n} d m\left(a_{n}\right),
$$

then since $E S_{a_{n}}(|x|) \leq \frac{1}{b}\|x\|_{1}$, for some $b>1$ such that $0<a_{n} \leq 1 \leq b$, while $\int_{0}^{1} a_{n} d m\left(a_{n}\right)=1$, for any $n \in \mathbb{N}$, hence there is some $c>1 \geq a_{n}>0$ for any $n$, such that

$$
E S_{a_{n}}(x) \leq \frac{1}{b \cdot c}\|x\|_{1},
$$

for any $n \in \mathbb{N}$. The last inequality implies

$$
\rho(x) \leq \frac{1}{b \cdot c}\|x\|_{1}
$$

which completes the proof.

Theorem 24. Any Kusuoka Representable risk measure on $L^{1}$ is strongly sensitive.

Proof: By [18, Pr.1], any such risk measure $\rho$ is a coherent risk measure on $L^{1}$, which admits the representation

$$
\rho(Z)=\sup _{\mu \in \overline{\mathcal{M}}} \int_{0}^{1} E S_{a}(Z) d \mu(a),
$$

where $Z \in L^{1}$ and $\overline{\mathcal{M}}$ denotes the closure under weak topology of a set of probability measures $\mathcal{M}$ on $[0,1]$. More specifically,

$$
|\rho(|Z|)| \leq \int_{0}^{1} E S_{a}(|Z|) d \mu(a) \leq \frac{1}{b}\|Z\|_{1},
$$

for some $b>1$ such that $0<a \leq 1<b$, which implies strong sensitivity.

Theorem 25. The pointwise limit of a sequence of Kusuoka Representable risk measures on $L^{1}$ is a strongly sensitive coherent risk measure.

Proof: By [18, Pr.1], any such risk measure $\rho_{n}$ of the sequence $\left(\rho_{n}\right)_{n \in \mathbb{N}}$ is a coherent risk measure on $L^{1}$, which admits the representation

$$
\rho_{n}(Z)=\sup _{\mu \in \overline{\mathcal{M}_{n}}} \int_{0}^{1} E S_{a}(Z) d \mu(a),
$$

where $Z \in L^{1}$ and $\overline{\mathcal{M}_{n}}$ denotes the closure under weak topology of a set of probability measures $\mathcal{M}_{n}$ on $[0,1]$. More specifically,

$$
\left|\rho_{n}(Z)\right| \leq \int_{0}^{1} E S_{a}(|Z|) d \mu(a) \leq \frac{1}{b}\|Z\|_{1},
$$


for some $b>1$ such that $0<a \leq 1<b$. Since $\rho_{n}(Z) \rightarrow \rho(Z)$, for the same $b>1$, we get $|\rho(|Z|)| \geq \frac{1}{b}\|Z\|_{1}$. WWe may quote on the coherence of poitwise limits of a sequence of coherent risk measures -being defined for example on $L^{1}$ - at this point.

Proposition 26. The pointwise limit $\rho$ of a sequence of coherent risk measures $\left(\rho_{n}\right)_{n \in \mathbb{N}}: L^{1} \rightarrow \mathbb{R}$, is a coherent risk measure on $L^{1}$.

Proof: It suffices to prove that $\rho$ satisfies the four properties of coherence. $\rho_{n}(Z+a \mathbf{1})=\rho_{n}(Z)-a, Z \in L^{1}, a \in \mathbb{R}$ denotes the Translation Invariace of $\rho_{n}, n \in \mathbb{N}$. From the uniqueness of the limit of the sequence of real numbers $\left(\rho_{n}(Z+a \mathbf{1})\right)_{n \in \mathbb{N}}$, which is $\rho(Z+a \mathbf{1})$ is equal to $\rho(Z)-a$. By the same way we deduce the Positive Homogeneity of $\rho$. About Subadditivity of $\rho$, we notice that for any $x, y \in L^{1}$ and $n \in \mathbb{N}$, the inequality

$$
\rho_{n}(x+y)-\rho_{n}(x)-\rho_{n}(y) \leq 0,
$$

holds in the set on real numbers. This implies that for the limit of this sequence

$$
\rho(x+y)-\rho(x)-\rho(y),
$$

the same inequality is true. Finally, if $x \geq y, \mathbb{P}-$ a.e. for any $n \in \mathbb{N}$, the inequality

$$
\rho_{n}(x)-\rho_{n}(y) \leq 0,
$$

holds in the set of real numbers. This implies that for the limit $\rho(x)-\rho(y)$, the same inequality holds.

About the notion of strong sensitivity of risk measures, see [14].

Proposition 27. If $\rho: L^{1} \rightarrow \mathbb{R}$ is strongly sensitive, then the associated performance ratio $a_{\text {rho,f }}: L^{1} \rightarrow \mathbb{R}$, where $a_{\rho, f}(x)=\frac{f(x)}{\rho(x)}$ and $f \in L_{+}^{\infty}$ being a strictly positive functional of $L^{1}$, is strongly sensitive.

Proof: If $\rho$ is strongly sensitive, this implies that if $x=y, \mu$, a.e. this implies $\rho(x)=\rho(y)$. But if $x=y$, a a.e. this implies $f(x-y)=0, f(y-x)=0$, since $f$ is strictly positive. This implies $a_{\rho, f}(x)=a_{\rho, f}(y)$.

Corollary 28. The performance ratio $\operatorname{ESR}_{t}: L^{1} \rightarrow \mathbb{R}$, where $\operatorname{ESR}_{t}(x)=$ $\frac{\mathbb{E}(x)}{E S_{a}(x)}$, is strongly sensitive.

Proof: It arises from the Proposition 27.

Corollary 29. The performance ratio $S R_{\phi}: L^{1} \rightarrow \mathbb{R}$, where $S R_{\phi}(x)=\frac{\mathbb{E}(x)}{\operatorname{phi}(x)}$ and $\phi$ is a spectral risk measure, is strongly sensitive.

Proof: It arises from the Proposition 27. 


\section{Optimization of Performance Ratios}

The general static optimization problem for some performance ratio $a_{\rho, f}$ is the following:

$$
\text { Maximize } a_{\rho, f}(x) \text { s.t. } x \in S,
$$

where $S$ is a proper subset of some normed linear space $E$.

Theorem 30. If E has a well-based cone $K$, and for the constraints' set that

$$
S \cap\left(-\operatorname{int}\left(K_{n}\right)\right)=\emptyset
$$

where $K_{n}, n \geq 2$ is some of the Henig Dilating Cones, (see Appendix 6.2), then the problem takes the form

$$
\text { Maximize } a_{\rho, f}(x)-z_{S}(x) \text { s.t. } x \in E,
$$

where $z_{S}$ is continuous, convex.

Proof: We apply the Separation Theorem [6, Th.2.3.6]. Namely, we pose $A=S, D=K_{n}, n \geq 2$. Also, we take some $k^{0} \in K_{n+2}, k^{0} \neq 0$, and according to the same Lemma $C=K_{n+2}$, being a cone, satisfying the condition $C+\operatorname{int}(D) \subseteq C$. Hence, the conclusion of [6, Th.2.3.6], (since the recession conditions $2.22,2.28$ also hold for $K_{n}, k^{0}$ ), implies the existence of a continuous, convex functional $z_{K_{n}, k^{0}}$, such that the scalarization of the constraint of the above problem, as follows:

$$
\text { Maximize } a_{\rho, f}(x) \text { s.t. } z_{K_{n}}(x) \geq 0 \text {, }
$$

where $z_{S}: E \rightarrow \mathbb{R}$ is the continuous, convex functional separating $S$ and $-\operatorname{int}\left(K_{n}\right)$. If we would like to transfer the scalar constraint itself inside the objective function, the problem becomes

$$
\text { Maximize } a_{\rho, f}(x)-z_{K_{n}, k^{0}}(x) \text { s.t. } x \in E,
$$

namely an unconstrained maximization problem of some continuous, concave functional. By following [6], $z_{K_{n}, k^{0}}(x)=\inf \left\{t \in \mathbb{R} \mid x \in t k^{0}-K_{n}\right\}$.

Theorem 31. The functional $\left.z_{K_{n}}, k^{0}\right)$ is a coherent risk measure with respect to the pair $\left(-K_{n},-k^{0}\right)$. The dual representation of $z_{K_{n}, k^{0}}$ is

$$
z_{K_{n}, k^{0}}(x)=\sup _{\pi \in \Pi} \pi(-x)
$$

where $\Pi=\left\{y \in \mathcal{A}_{\rho_{\left(-K_{n},-k^{0}\right)}}^{0} \mid\left(-k^{0}\right)(y)=1\right\}$. The set $\mathcal{A}_{\rho_{\left(-K_{n},-k^{0}\right)}}^{0}$ denotes the polar wedge of the acceptance set $\mathcal{A}_{\rho_{\left(-K_{n},-k^{0}\right)}}$ of $\left.\rho_{\left(-K_{n},-k^{0}\right)}=z_{K_{n}}, k^{0}\right)$. 
Proof: According to [13, Lem.3.2, Cor.3.3, Th.3.4], $z_{K_{n}, k^{0}}(x)=\inf \{t \in$ $\left.\mathbb{R} \mid y \in t k^{0}-K_{n}\right\}==\inf \left\{t \in \mathbb{R} \mid x+t\left(-k^{0}\right) \in-K_{n}\right\}=\rho_{\left(-K_{n},-k^{0}\right)}(x)$. Hence, $\left(-K_{n}\right) \subseteq \mathcal{A}_{\rho_{\left(-K_{n},-k^{0}\right)}}$ and for the corresponding polar wedges, $\mathcal{A}_{\rho_{\left(-K_{n},-k^{0}\right)}} \subseteq$ $\left(-K_{n}\right)^{0}$. The cone $\left(-K_{n}\right)^{0}$ is the ordering cone in $E^{*}$, and $\left(-k^{0}\right)$ defines a closed, bounded base on it. $\Pi$ is the part of it, which belongs to the polar $\mathcal{A}_{\rho_{\left(-K_{n}, k^{0}\right)}}^{0}$ of the acceptance set.

It is well known that the solution of a maximization problem of a concave function, which in this case is $a_{\rho, f}(x)-z_{K_{n}, k^{0}}(x)$ over the entire space $E$ is equivalent to the solution of the minimization problem of the convex function

$$
g(x)=z_{K_{n}, k^{0}}(x)-a_{\rho, f}(x)
$$

all over $E$. For this reason we would like to determine these $\bar{x} \in E$, such that $0 \in \partial g(\bar{x})$, where $\partial g \subseteq E^{*}$ denotes the subdifferential correspondence of $g$, see [19, Th.2.5.7].

We notice that in $E=L^{2}$, the product $x y$ of two elements $x, y$ of $L^{2}$ is an element of $L^{2}$.

Theorem 32. In $E=L^{2}$, the above optimality condition $0 \in \partial g$ becomes $\pi_{x}=\pi_{y}$ for any $x, y \in E$, if the representation set $D$ of the coherent risk measure $\rho$ of $a=a_{\rho, f}$ is weakly compact.

Proof: For any $\pi \in \Pi$ and if $D$ is weakly compact, we have

$$
\begin{aligned}
g(x)-g(y) \geq & \pi(y-x)-a(x)+a(y) \geq\left(\pi(-x)-\frac{f(x)}{\pi_{x}(-x)}\right)-\left(\pi(-y)-\frac{f(y)}{\pi_{y}(-y)}\right)= \\
& =\left(-\pi(x)+\frac{f(x)}{\pi_{x}(x)}\right)-\left(-\pi(y)+\frac{f(y)}{\pi_{y}(y)}\right)=\left\langle x-y, x^{*}\right\rangle .
\end{aligned}
$$

Then, $x^{*}=\pi+\frac{f}{\pi_{x}}=\pi+\frac{f}{\pi_{y}}$ is the optimality condition, which becomes $\pi_{x}=\pi_{y}$ for any $x, y \in E$.

\section{References}

[1] C. Acerbi, Spectral measures of risk: A coherent representation of subjective risk aversion, Journal of Banking and Finance, 26 (2002), 1505-1518. http://dx.doi.org/10.1016/s0378-4266(02)00281-9

[2] C.D. Aliprantis and K. C. Border, Infinite Dimensional Analysis: A Hitchhiker's Guide, 3rd Ed.: Springer, Berlin Heidelberg, 2006.

http://dx.doi.org/10.1007/3-540-29587-9 
[3] F. Bellini and V. Bignozzi, On Elicitable Risk Measures, Quantitative Finance, 15 (2015), 725-733.

http://dx.doi.org/10.1080/14697688.2014.946955

[4] A. Cherny and D. Madan, New Measures for Performance Evaluation, Review of Financial Studies, 22 (2009), 2571-2606.

http://dx.doi.org/10.1093/rfs/hhn081

[5] X.H. Gong, Connectedness of efficient solution sets for set-valued maps in normed spaces, Journal of Optimization Theory and Applications, 83 (1994), 83-96. http://dx.doi.org/10.1007/bf02191763

[6] A. Göpfert, H. Riahi, C. Tammer, C. Zălinescu, Variational Methods in Partially Ordered Spaces, CMS Books in Mathematics, Springer, New York, 2003. http://dx.doi.org/10.1007/b97568

[7] S. Farinelli et al., Beyond Sharpe Ratio: Optimal Asset Allocation Using Different Performance Ratios, Journal of Banking and Finance, 32 (2008), 2057-2063. http://dx.doi.org/10.1016/j.jbankfin.2007.12.026

[8] H. Föllmer and A. Schied, Convex measures of risk and trading constraints, Finance and Stochastics, 6 (2002), 429-447.

http://dx.doi.org/10.1007/s007800200072

[9] H. Föllmer, Spatial risk measures and their local specification: The local law-invariant case, Stat. Risk Modeling, 31 (2014), 79-101.

http://dx.doi.org/10.1515/strm-2013-5001

[10] A. Inoue, On the worst conditional expectation, Journal of Mathematical Analysis and Applications, 286 (2003), 237-247.

http://dx.doi.org/10.1016/s0022-247x(03)00477-3

[11] M. Kaina and L. Rüschendorf, On Convex Risk Measures on $L^{p}$ spaces, Mathematical Methods of Operations Research, 69 (2009), 475-495.

http://dx.doi.org/10.1007/s00186-008-0248-3

[12] V.N. Katsikis and I.A. Polyrakis, Positive bases in ordered subspaces with the Riesz decomposition property, Studia Mathematica, 174 (2006), 233253. http://dx.doi.org/10.4064/sm174-3-2

[13] C.E. Kountzakis, Risk measures on ordered non-reflexive Banach spaces, Journal of Mathematical Analysis and Applications, 373 (2011), 548-562. http://dx.doi.org/10.1016/j.jmaa.2010.08.013

[14] C.E. Kountzakis, Sensitivity of Risk Measures defined on $L^{1}$-spaces, To appear on Applied Mathematical Sciences. 
[15] C. Kountzakis and I.A. Polyrakis, Coherent risk measures in general economic models and price bubbles, Journal of Mathematical Economics, 49 (2013), 201-209. http://dx.doi.org/10.1016/j.jmateco.2013.02.002

[16] D.G. Konstantinides and C.E. Kountzakis, Coherent Risk Measures under Dominated Variation, Chapter in Modern Problems in Insurance Mathematics (International Cramér Symposium on Insurance Mathematics), (Eds) D. Silvestrov, A.M. Löf, Springer International Publishing, 2013, 113-138. http://dx.doi.org/10.1007/978-3-319-06653-0_8

[17] S. Kusuoka, On Law -Invariant Coherent Risk Measures, Chapter in Advances in Mathematical Economics, (Eds) S. Kusuoka, T. Maruyama, Springer, 2001, 83-95. http://dx.doi.org/10.1007/978-4-431-67891-5_4

[18] A. Shapiro, On Kusuoka Representation of Law Invariant Risk Measures, Mathematics of Operations Research, 38 (2013), 142-152.

http://dx.doi.org/10.1287/moor.1120.0563

[19] C. Zălinescu, Convex Analysis in General Vector Spaces, World Scientific, 2002. http://dx.doi.org/10.1142/9789812777096

\section{Appendix}

\subsection{The compactness of the representation set of $E S_{a}$}

Lemma 33. $\left\{\frac{d Q}{d \mathbb{P}} \mid Q \in c a(\Omega), Q(\Omega)=1, Q<<\mathbb{P}, \frac{d Q}{d \mathbb{P}} \in\left[0, \frac{1}{a}\right], \mathbb{P}-\right.$ a.e. $\}$ is a weak-star compact set of $L^{\infty}(\Omega, \mathcal{F}, \mathbb{P})$.

Proof:

$$
\mathcal{Z}_{a}=\left\{\frac{d Q}{d \mathbb{P}} \mid Q \in c a(\Omega), Q(\Omega)=1, Q<<\mathbb{P}, \frac{d Q}{d \mathbb{P}} \in\left[0, \frac{1}{a}\right], \mathbb{P}-\text { a.e. }\right\} .
$$

and the order-interval $\left[0, \frac{1}{a} \mathbf{1}\right]$ of $L^{\infty}$ is $\sigma\left(L^{\infty}, L^{1}\right)$-compact due to [2, Th.8.60]. We also have to prove that $Z_{a}$ is weak-star closed in $L^{\infty}$. Let us consider a net $\left(Q_{\lambda}\right)_{\lambda \in \Lambda} \subseteq Z_{a}$ such that

$$
\frac{d Q_{\lambda}}{d \mathbb{P}} \stackrel{\sigma\left(L^{\infty}, L^{1}\right)}{\rightarrow} f
$$

From the fact that $\frac{d Q_{\lambda}}{d \mathbb{P}}, \lambda \in \Lambda$, we obtain that $\frac{d Q_{\lambda}}{d \mathbb{P}} \in L^{1}(\Omega, \mathcal{F}, \mathbb{P})$. We have to prove that $f$ is a Radon-Nikodym derivative of some measure $Q_{1} \in \mathcal{Z}_{a}$ with respect to $\mathbb{P}$. Let us consider the map $Q_{1}: \mathcal{F} \rightarrow[0,1]$ where

$$
Q_{1}(A)=\int_{A} f \cdot I_{A} d \mathbb{P}
$$


and $I_{A}$ is the characteristic random variable of $A$. In order to show that $Q_{1}$ is a probability measure,

$$
Q_{1}(\Omega)=\int_{\Omega} f d \mathbb{P}
$$

which is the limit $\lim _{\lambda \in \Lambda} \int_{\Omega} d Q_{\lambda}$ and every of the terms of the net of real numbers

$$
\left(\int_{\Omega} d Q_{\lambda}\right)_{\lambda \in \Lambda}
$$

is equal to 1 . By the same argument, we may deduce that $Q_{1}(\emptyset)=0$. If $\left(A_{n}\right)_{n \in \mathbb{N}}$ is a sequence of sets in $\mathcal{F}$ which are disjoint, then

$$
Q_{\lambda}\left(\cup_{k=1}^{n} A_{k}\right)=\sum_{k=1}^{n} Q_{\lambda}\left(A_{k}\right), \lambda \in \Lambda
$$

Hence,

$$
Q_{1}\left(\cup_{k=1}^{n} A_{k}\right)=\sum_{k=1}^{n} Q_{1}\left(A_{k}\right), n \in \mathbb{N},
$$

where $\mathbb{N}$ denotes the set of natural numbers. For $n \rightarrow \infty$

$$
Q_{1}\left(\cup_{n=1}^{\infty} A_{n}\right)=\sum_{n=1}^{\infty} Q_{1}\left(A_{n}\right),
$$

from the $\sigma\left(L^{\infty}, L^{1}\right)$-convergence

$$
\frac{d Q_{\lambda}}{d \mathbb{P}} \stackrel{\sigma\left(L^{\infty}, L^{1}\right)}{\rightarrow} f
$$

and the definition of $Q_{1}$, the fact that any characteristic function $I_{A}, A \in$ $\mathcal{F}$ belongs to $L^{1}(\Omega, \mathcal{F}, \mathbb{P})$. We may also refer to the Monotone Convergence Theorem [2, Th.11.18], where the restriction of the $Q_{1}$ on the set $\cup_{n=1}^{\infty} A_{n}$ is the integrable function which is mentioned in the Theorem, while $f_{n}$ is the restriction of $Q_{1}$ on a set of the form $\cup_{k=1}^{n} A_{k}$. For the $\mathbb{P}$-continuity of $Q_{1}$, we have that if for a set $A \in \mathcal{F} \mathbb{P}(A)=0$ holds, then since $Q_{\lambda}, \lambda \in \Lambda$ is $\mathbb{P}$-continuous,

$$
Q_{\lambda}(A)=0=\int_{A} \frac{d Q_{\lambda}}{d \mathbb{P}} d \mathbb{P}
$$

for any $\lambda \in \Lambda$. But since

$$
\frac{d Q_{\lambda}}{d \mathbb{P}} \stackrel{\sigma\left(L^{\infty}, L^{1}\right)}{\rightarrow} f
$$

then

$$
Q_{1}(A)=\int_{A} f d \mathbb{P}=\lim _{\lambda \in \Lambda} \int_{A} \frac{d Q_{\lambda}}{d \mathbb{P}} d \mathbb{P}=0 .
$$


Hence $Q_{1}$ is $\mathbb{P}$-continuous. Since $Q_{\lambda}, \lambda \in \Lambda$ are probability measures,

$$
\frac{d Q_{\lambda}}{d \mathbb{P}}(\omega) \geq 0
$$

$\mathbb{P}$-a.e. Also, since $Q_{1}$ is a $\mathbb{P}$-continuous probability measure, by Radon-Nikodym Theorem we have

$$
\frac{d Q_{1}}{d \mathbb{P}}=f
$$

$\mathbb{P}$-a.e. and $f(\omega) \geq 0, \mathbb{P}$-a.e. In order to show that

$$
0 \leq f \leq \frac{1}{a} \mathbf{1}
$$

with respect to the usual (point-wise) partial ordering on $L^{\infty}(\Omega, \mathcal{F}, \mathbb{P})$, we use the convergence argument

$$
\int_{A} \frac{d Q_{\lambda}}{d \mathbb{P}} d \mathbb{P} \rightarrow \int_{A} f d \mathbb{P},
$$

for any $A \in \mathcal{F}$. This implies that $\int_{A} f d \mathbb{P} \in\left[0, \frac{1}{a}\right]$ for any $A \in \mathcal{F}$. This implies $0 \leq f \leq \frac{1}{a} \mathbf{1} \mathbb{P}$-a.e., since if we suppose that this does not hold, then there exists some $B \in \mathcal{F}$ with $\mathbb{P}(B)>0$ such that either $f(\omega)>\frac{1}{a}$, or $f(\omega)<0$ for any $\omega \in B$. Then, we would have either $\int_{B} f d \mathbb{P}>\frac{1}{a}$, or $\int_{B} f d \mathbb{P}<0$, a contradiction. Finally, the set $\mathcal{Z}_{a}$ is a weak-star closed subset of a weak-star compact set which is the set $\mathcal{D}_{a}$.

\subsection{Henig Dilating Cones}

We remind of $[5$, Lem.2.1]

Lemma 34. If the closed cone $K$ has a base $B$, such that $0 \notin \bar{B}$, we define

$$
K_{n}=\overline{c o n e}\left(B+\frac{\delta}{n} B(0,1)\right)
$$

where $B(0,1)$ is the closed unit ball of $E$ and $\delta>0$, such that $2 \delta B(0,1) \cap B=\emptyset$, namely $\|b\|>2 \delta, b \in B$ and $0 \notin \overline{B+\delta B(0,1)}$. Then, the following are true:

(i) $K \subseteq K_{n+1} \subseteq K_{n}, n \geq 1$

(ii) $K_{n+1} \subseteq \operatorname{cone}\left(B+\frac{\delta}{n} B(0,1)\right), n \geq 1$.

(iii) $K \backslash\{0\} \subseteq \operatorname{int}\left(K_{n}\right), n \geq 1$.

(iv) $K_{n}$ is a cone $n \geq 2$.

Proof: 
(i) Obvious.

(ii) Let $n$ a specific one and $y \in K_{n+1} \backslash K$. Then, there exists a sequence $\left\{y_{m}\right\} \subseteq \operatorname{cone}\left(B+\frac{1}{n+1} \delta B(0,1)\right)$, such that $y_{m} \rightarrow y$, such that $y_{m}=$ $\lambda_{m}\left(b_{m}+\frac{\delta}{n+1} u_{m}\right), u_{m} \in B(0,1), b_{m} \in B, \lambda_{m}>0$. Let $\epsilon_{0}>0$, such that $\lambda_{m} \geq \epsilon_{0}$, for any $m$. We select a $\epsilon: 0<\epsilon<\frac{\epsilon_{0}}{(n+1)^{2}}$ and we notice that

$$
\frac{\epsilon}{\lambda_{m}}<\frac{\epsilon}{\lambda_{m}(n+1)^{2}} \leq \frac{\epsilon_{0}}{(n+1)^{2} \epsilon_{0}}=\frac{1}{(n+1)^{2}}
$$

for any $m$. Since $y_{m} \rightarrow y$, there exists $m_{0}$ such that

$$
\begin{gathered}
y \in y_{m_{0}}+\epsilon \delta B(0,1)=\lambda_{m_{0}}\left(b_{m}+\frac{\delta}{n+1} u_{m_{0}}\right)+\epsilon \delta B(0,1)= \\
\left.\lambda_{m_{0}}\left(b_{m_{0}}+\frac{\delta}{n+1} u_{m_{0}}\right)+\frac{\epsilon}{\lambda_{m_{0}}} \delta B(0,1)\right) \\
\left.\subseteq \lambda_{m_{0}}\left(b_{m_{0}}+\frac{\delta}{n+1} u_{m_{0}}\right)+\frac{1}{(n+1)^{2}} \delta B(0,1)\right) \\
\subseteq \lambda_{m_{0}}\left(b_{m_{0}}+\frac{1}{n} \delta B(0,1)\right) \subseteq \operatorname{cone}\left(B+\frac{1}{n} \delta B(0,1)\right) .
\end{gathered}
$$

(iii) We will show that

$$
\operatorname{cone}\left(B+\frac{\delta}{n+1} B(0,1)\right) \backslash\{0\} \subseteq \operatorname{int}\left(K_{n}\right), n \geq 1 .
$$

Let $y \in \operatorname{cone}\left(B+\frac{\delta}{n+1} B(0,1)\right) \backslash\{0\}$. Then, there exist $\lambda>0, b \in B, u \in$ $B(0,1)$, such that $y=\lambda\left(b+\frac{\delta}{n+1} u\right)$. Then we will have that

$$
\begin{gathered}
y+\frac{\lambda \delta}{(n+1)^{2}} B(0,1)=\lambda\left(b+\frac{\delta}{n+1} u\right)+\frac{\lambda \delta}{(n+1)^{2}} B(0,1)= \\
\lambda\left(b+\frac{\delta}{n+1} u\right)+\frac{\lambda \delta}{(n+1)^{2}} B(0,1) \subseteq \lambda\left(b+\frac{\delta}{n} B(0,1)\right) \subseteq \operatorname{cone}\left(B+\frac{\delta}{n} B(0,1)\right) .
\end{gathered}
$$

Since $y+\frac{\lambda \delta}{(n+1)^{2}} B(0,1)$ is an open neighborhood of $y, y \in \operatorname{int}(\operatorname{cone}(B+$ $\left.\left.\frac{\delta}{n} B(0,1)\right)\right)$. Then,

$$
\begin{gathered}
K \backslash\{0\} \subseteq K_{n+2} \backslash\{0\} \subseteq \operatorname{cone}\left(B+\frac{\delta}{n+1} B(0,1)\right) \backslash\{0\} \\
\subseteq \operatorname{int}\left(\operatorname{cone}\left(B+\frac{\delta}{n} B(0,1)\right)\right) \subseteq \operatorname{int}\left(K_{n}\right), n \geq 1
\end{gathered}
$$


(iv) The set $\overline{B+\delta B(0,1)}$ is closed, convex and $0 \notin \overline{B+\delta B(0,1)}$. Separating in the strict sense these sets by an element $h \in E^{*}$, we notice that $h(y)>0, y \in \operatorname{cone}(B+\delta B(0,1)) \backslash\{0\}$. By $(i),(i i)$ we have $K_{n} \backslash\{0\} \subseteq K_{2} \backslash\{0\} \subseteq \operatorname{cone}(B+\delta B(0,1)) \backslash\{0\} \subseteq\{y \in E: h(y)>0\}$. Hence, $K_{n}$ is a cone, if $n \geq 2$.

Received: July 6, 2016; Published: September 2, 2016 